\title{
Spatial distribution of coda $Q$ estimated from local earthquakes in Taiwan area
}

\author{
Jen-Kuang Chung ${ }^{1}$, Yen-Ling Chen ${ }^{2,3}$, and Tzay-Chyn Shin $^{3}$ \\ ${ }^{1}$ Institute of Geoinformatics and Disaster Reduction Technology, Ching Yun University of Technology, Taiwan \\ ${ }^{2}$ Institute of Geosciences, National Taiwan University, Taiwan \\ ${ }^{3}$ Central Weather Bureau, Taiwan
}

(Received December 17, 2008; Revised April 8, 2009; Accepted June 1, 2009; Online published November 10, 2009)

\begin{abstract}
Attenuation of seismic wave energy and its spatial distributions in Taiwan area have been investigated using a large amount of short-period seismograms recorded from the local earthquakes with magnitude ranging from 2.5 to 4.5. The coda $Q$ values were estimated in the frequency range $1.5 \sim 18 \mathrm{~Hz}$, applying the single backscattering model proposed by Aki and Chouet in 1975. The estimated average frequency dependent coda $Q$ values within the crust give the relationship, $Q_{C}=93 f^{0.75}$, while varying from $143 \pm 33$ at $1.5 \mathrm{~Hz}$ to $904 \pm 164$ at $18 \mathrm{~Hz}$ central frequencies. However, using the data from deeper earthquakes, the average coda $Q$ relationship is given as $Q_{C}=122 f^{0.71}$ in related to the material property of the crust and upper lithosphere, in which the average coda $Q$ values vary from $180 \pm 67$ at $1.5 \mathrm{~Hz}$ to $1000 \pm 144$ at $18 \mathrm{~Hz}$. The comparison of different lapse times starting at double the time of the primary $S$-wave from the origin time and corresponding to the datasets with different focal depths, reveals that coda $Q$ values increase with the coda generating volume. As a whole, the regressive relationships of frequency dependent coda $Q$ for Taiwan region approximately agree with those by previous investigators. In addition, the average coda $Q$ values for three subregions of Taiwan were calculated and correlated with the geology and tectonic features, respectively. The lowest $Q$ values were obtained in the region consisting of the Coastal Range and the Longitudinal Valley of eastern Taiwan, whilst the highest $Q$ for the region underlain by the Peikang High beneath the western Coastal Plain. Such distinguishable difference has definitely explained that the seismic waves should exhibit lower decay rate in a stable region than within a seismoactive plate boundary.
\end{abstract}

Key words: Coda $Q$, scattering, lapse time, Taiwan, attenuation.

\section{Introduction}

Attenuation of seismic waves propagating through a realistic medium plays an important role on the prediction of ground motion induced by an earthquake. The characteristics of seismic coda waves are generally described by the average decay of the envelope in the tail portion of a seismogram (Aki, 1969). Based on the concept that the earth is assumed to be composed by the randomly distributed heterogeneities, the behavior of coda waves can be adequately explained by the scattering of primary elastic waves in a random medium. The first attempt to predict the explicit form of time-dependent power spectrum of coda waves was made by Aki and Chouet (1975) assuming that coda waves are singly back-scattered $S$ waves. A generalization form of Aki and Chouet's model were proposed by Sato (1977). In this model the scattering is a weak process, and use is made of the Born approximation in that the loss of energy from the primary waves as well as the multiple scattering is neglected in the original idea. Putting the controversy on the mechanism of energy dissipation aside, the contention of scattered $S$ to $S$ waves from heterogeneities in the crust and the upper mantle can be widely supported by the work of Rautian and Khalturin (1978), Aki (1980), Herrmann

Copyright (c) The Society of Geomagnetism and Earth, Planetary and Space Sciences (SGEPSS); The Seismological Society of Japan; The Volcanological Society of Japan; The Geodetic Society of Japan; The Japanese Society for Planetary Sciences; TERRAPUB
(1980), Roecker et al. (1982) and Sarker and Abers (1998) who have revealed that the coda $Q\left(Q_{C}\right)$ and the shear-wave $Q\left(Q_{S}\right)$ are similar for the studied regions.

Gao et al. (1983) investigated the effects of multiple scattering on coda waves from a uniform distribution of isotropic scatterers in an elastic medium by an extension of the single scattering theory. Their results suggested that at shorter lapse time measured from the origin time of event, the coda power is well explained by the single scattering theory, whilst the effects of multiple scattering need to be taken into account for longer ( $>100 \mathrm{~s}$ ) lapse time. Conversely, Sato (1988) demonstrated that the single scattering model is valid even for the long lapse time. On the other hand, based on an energy-flux model, Frankel and Wennerberg (1987) suggested that coda $Q$ can be attributed to the intrinsic loss demonstrated by their finite difference simulations. The idea is that energy is uniformly distributed within some region surrounding an earthquake at some lapse time. This model results in a simple formula which explicitly differentiates between the scattering and anelastic attenuation of the medium, and is valid for both weak and strong scattering. Applying the radiative transfer theory Wu (1985) proposed a method to estimate the relative contribution of scattering and intrinsic loss to the total attenuation. For providing a complete analysis of scattered wave energy, Zeng et al. (1991) extended the stationary energy transport theory to the time dependent case and obtained the scattered 
wave energy equation, which can be solved by applying the Fourier transform technique and the feasible numerical procedures, such as a Monte Carlo simulation. Sato (1994) also proposed a hybrid technique combining the analytical solutions corresponding to single scattering and numerical calculations for multiple scattering to obtain better explanations for the effect of attenuation on different seismic waves. Gusev (1995) showed that coda decay is quantitatively well explained if the total scattering coefficient decreases with depth, when the leakage of scattered energy to the bottom cannot be discriminated from intrinsic loss. For obtaining reasonable interpretations of well known lapsetime dependence of the coda $Q$ (Roecker et al., 1982), the models incorporating non-uniform distribution of scatterers and the possibility that intrinsic dissipation may decrease with depth could be more appropriate than those assuming a uniform earth model. However, there is no simple relation between coda $Q$ and scattering and intrinsic $Q$ (Aki, 1991; Mayeda et al., 1992). Although the radiative transfer approach (Wu, 1985) and the multiple lapse time window analysis method (Fehler et al., 1992) have been used for making estimates of the amount of scattering that is caused by intrinsic and scattering mechanisms, the practical problem in the multiple scattering model is having the relatively complicated procedures. The single backscattering methods, therefore, still seem to be most widely used due to their simplicity, particularly for small $(M<6)$ local earthquakes (Aki and Chouet, 1975; Rhea, 1984; Lee et al., 1986; Del Pezzo et al., 1990; Kumar et al., 2005; Jin and Aki, 2005; Yun et al., 2007).

Coda $Q$ varies with frequency, tectonic region, and the lapse time interval used in the observations. It has generally been found to be lower in tectonically seismic active regions and higher in seismic inactive stable regions (Singh and Herrmann, 1983; Jin and Aki, 1988, 2005; Canas et al., 1995). Also, Roecker et al. (1982) inferred that the variation between the initial and later decays of coda wave is primarily due to the coda $Q$ that varies with depth. This explanation, which has basically agreed with those by numerous researches, advise us have to take a depth-dependent scattering model theoretically and make a reasonable estimation of the volume sampled by coda waves from the used lapse time. The frequency dependence within a region (Mitchell, 1981) can be represented as $Q_{C}=Q_{0} f^{\eta}$ for $f>1 \mathrm{~Hz}$, where $Q_{0}$ is the $Q$ value at the reference frequency of $1 \mathrm{~Hz}$ and the frequency dependence factor $\eta$, which is indicative of the degree of heterogeneity of the crust (Aki, 1981), ranges between 0.1 and 1 for most worldwide regions.

With the superiority of long term monitoring of local earthquakes in Taiwan region by the seismic networks, the seismic anelastic attenuation has been investigated using short-period data (Shin et al., 1987; Wang, 1988; Chen et al., 1989) as well as strong motion records (Chang and Yeh, 1983), respectively, in the past. However, comparing the frequency-dependent attenuation relationships proposed by them, the $Q$ values for Taiwan area estimated from different types of waves and by various methods seem not compatible in the distribution patterns. Obvious discrepancy of $Q$ value was obtained between the western coastal area and the east of Taiwan, which are situated at different tectonic settings. A possible reason causing the different results will be the lack of enough good quality seismograms recorded at stations along the coast (Shin et al., 1987). Even with the high quality data, in practice, the variability of coda $Q$ for different stations and hypocenters is rather large in some cases. This may require appropriate averaging over a large number of stations and events to obtain a stable and accurate estimate of coda $Q$ (Lee et al., 1986; Hellweg et al., 1995; Giampiccolo et al., 2004; Tripathi and Ugalde, 2004).

In this paper, the attenuation properties of seismic coda waves observed in Taiwan region were investigated using the single backscattering method (Aki and Chouet, 1975) by analyzing a large number of short-period local seismograms. The spatial distribution of coda $Q$ will be of great interest to the seismic hazard assessment in the area. In substance, the aims of this paper are: i) investigate the $Q_{C}$ dependence on frequency, ii) map the spatial variations respectively on the free surface and along the depth, and iii) make a comparison with previous results for the area and correlate with the tectonic and geological features.

\section{Data and Method}

A digital seismic network, called CWBSN, operated by Central Weather Bureau, Taiwan, had been upgraded to a new modern earthquake monitoring system in 1991 for offering more precise earthquake locations in a higher efficiency. All the seventy-five seismic stations of this network are equipped with high quality velocity-type 3-component S-13 seismographs of Teledyne products, which have the capability of recording local inland earthquakes with smallest magnitude of $\sim 1.5$ as the trigger mode and continuous mode of recording data stream are parallel (Chang, 2004). The instruments were setup to digitize the signal at $100 \mathrm{sps}$ under the trigger mode of routine operation while $50 \mathrm{sps}$ in continuous mode based on the backup purpose for retrieving signals below the trigger level. In consequence of the great upgrade, the CWBSN records over 15,000 earthquakes in the Taiwan region every year, which is about triple the number of those recorded previously.

In this study, forty-five stations of this network were selected to perform a nearly uniform coverage over the island in an average spacing of about $25 \mathrm{~km}$ (Fig. 1). For each station we searched for earthquakes with local magnitude ranging from 2.5 to 4.5 located about $20 \mathrm{~km}$ from the station and with focal depth in the ranges of $2 \sim 15 \mathrm{~km}$ and $25 \sim 50 \mathrm{~km}$, respectively (Fig. 2). Few selected earthquakes occurred in northern Taiwan are exceptionally near to $60 \mathrm{~km}$ deep due to the lack of recordings from the required focal depth events. Most of earthquakes were randomly selected from the restricted dataset during the period from 1993 to 2006. During this period, the distribution of epicenters of earthquakes $\left(M_{\mathrm{L}} \geq 2.5\right)$ with focal depth less than $50 \mathrm{~km}$ is shown in Fig. 3. Wang and Shin (1998) have summarized some specific patterns of the seismicity. Following Aki (1969) the analysis is not sensitive to site effects, at least if a sufficiently large number of ray paths can be averaged. To avoid possible directivity effect, however, the epicenters in dataset were purposely categorized into eight sectors with different azimuth for the shallow earthquakes. Maximum of three recordings were arbitrarily cho- 


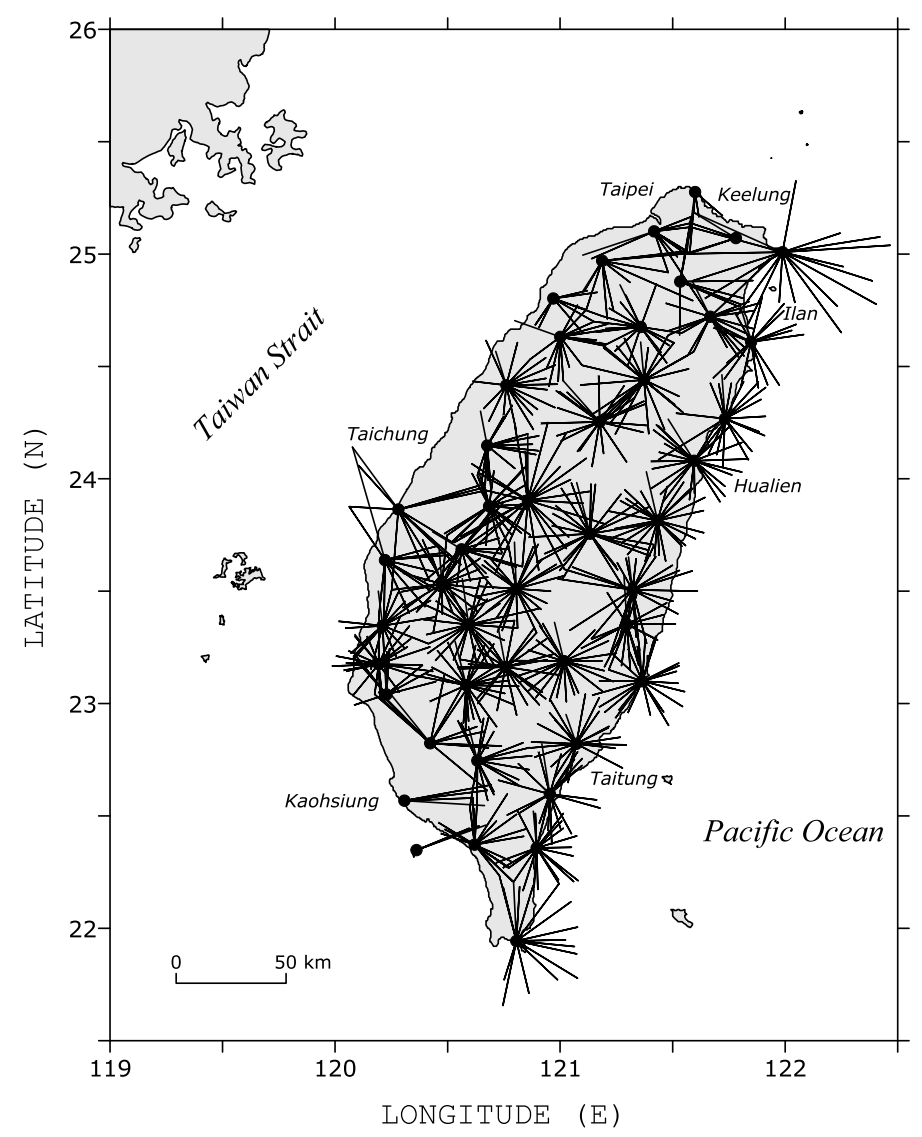

Fig. 1. S-13 stations (solid dots) of CWBSN and the projections of seismic-wave paths connecting the stations and the epicenters (not shown) of shallow earthquakes analyzed in this study.

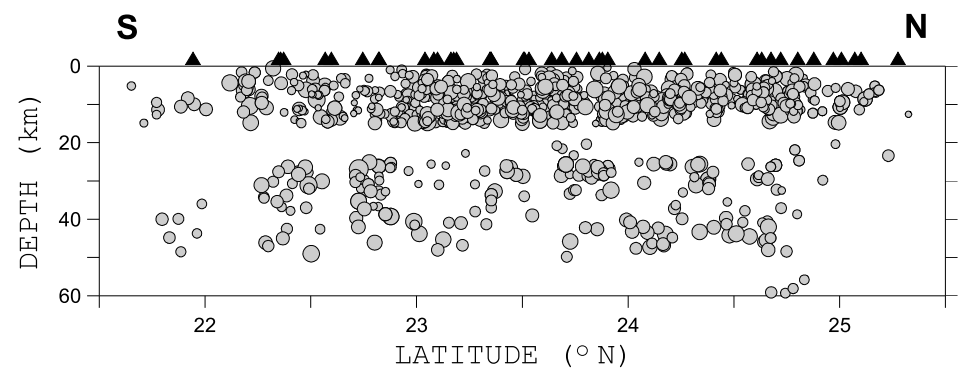

Fig. 2. Profile showing focal depths for analyzed events along the north-south direction. The radii of circles are proportional to local magnitude ranging from 2.5 to 4.5 . The used seismic stations are depicted by solid triangles.

sen in each sector with an azimuth range of $\pi / 4$ in radian. Thus, a set of 243 -component digital seismic waveforms collected from different directions roughly around each station were used for analyzing the average coda $Q$ value and the azimuth-dependent variation. For some stations, particularly close to the western coast as shown in Fig. 1, the poor azimuthal coverage of propagation path is realized because of low seismicity in the region from western coast to Taiwan Strait (Wang and Shin, 1998). On the other hand, only maximum of eight recordings from deep events were randomly selected without accounting for azimuthal distribution. The seismograms having the signal to noise ratio below 3 were discarded for obtaining reliable $Q_{C}$ values. Totally, 772 and 238 original 3-component seismograms from shallow events and deep ones, respectively, were analyzed herein. Both of the $P$ - and $S$-wave onsets on each seismogram were clearly depicted. Most of the earthquake locations are reliable with the error less than $3 \mathrm{~km}$ both in the coordinates of epicenter and the focal depth (Chang, 2004).

A most common technique developed to estimate the $Q$ values for coda waves in time domain, are essentially based on the fit of the seismogram's envelope, obtaining at different frequency bands, to the single backscattering model (Aki and Chouet, 1975). According to Aki and Chouet (1975), the rms amplitude of coda wave $A(f, t)$ in a seismogram, for a central frequency $f$ with a narrow bandwidth, and the lapse time $t$ measured from the origin time of seismic event, is given by:

$$
A(f, t)=A_{0}(f) t^{-\alpha} e^{-\pi f t / Q_{C}}
$$

where $Q_{C}$ is the quality factor of coda waves including the intrinsic and scattering attenuation of energy in the medium, 


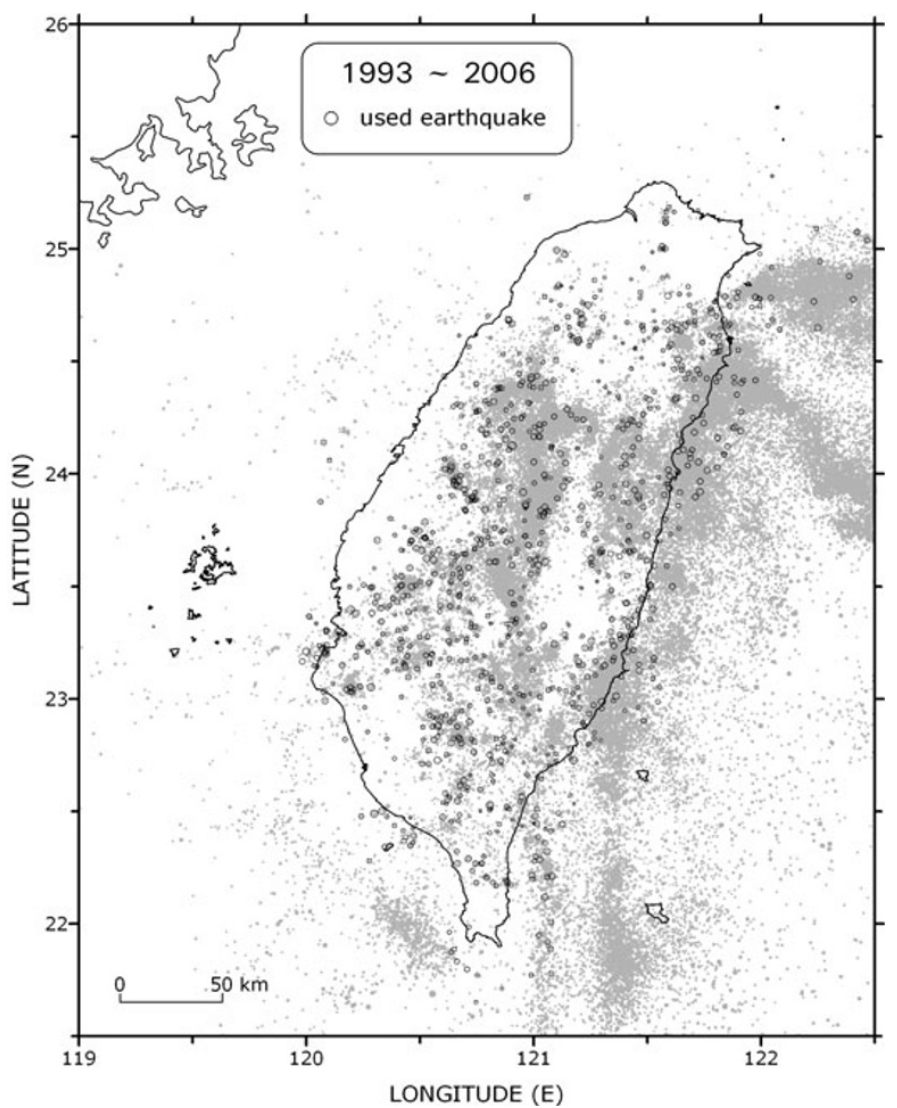

Fig. 3. Epicentral distribution of earthquakes (shown as gray dots) with local magnitude greater than 2.5 recorded during the period of $1993 \sim 2006$. The focal depths are less than $50 \mathrm{~km}$. The circles represent the earthquakes used in this study.

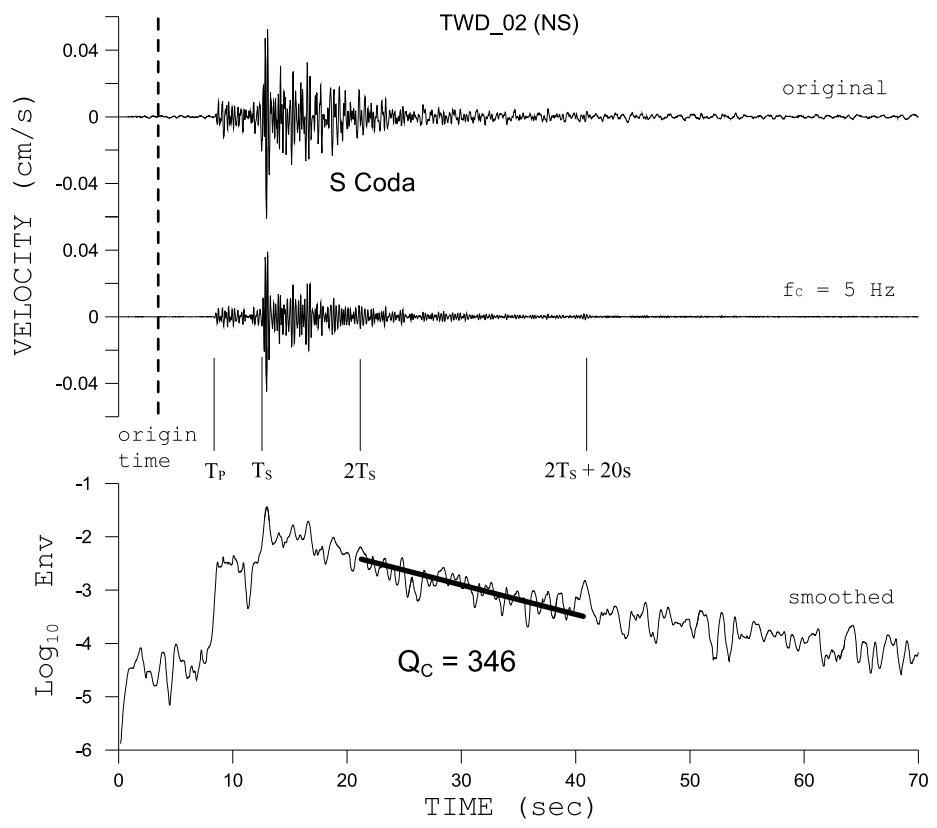

Fig. 4. An example of data processing for coda $Q$ estimation performed in this study. The top trace is one of NS-component velocity time histories recorded at station TWD located at Hualien. The middle trace is the bandpass-filtered record with a central frequency of $5 \mathrm{~Hz}$. The bottom trace shows the smoothed envelope of filtered record. The vertical dashed line indicates the origin time of earthquake. $T_{P}$ and $T_{S}$ represent the lapse times of $P$ - and $S$-arrivals, respectively.

$A_{0}(f)$ represents the coda source factor at frequency $f$, which is independent of time and radiation pattern, and $\alpha$ is a constant determined by the geometrical spreading, with $\alpha=0.5$ for surface waves and $\alpha=1$ for body waves, respectively. Taking logarithms of both sides of relationship (1), the $Q_{C}$ can be estimated by using a linear regression analysis. This approach can sufficiently describe the first-order phenomenology of coda decay according to 


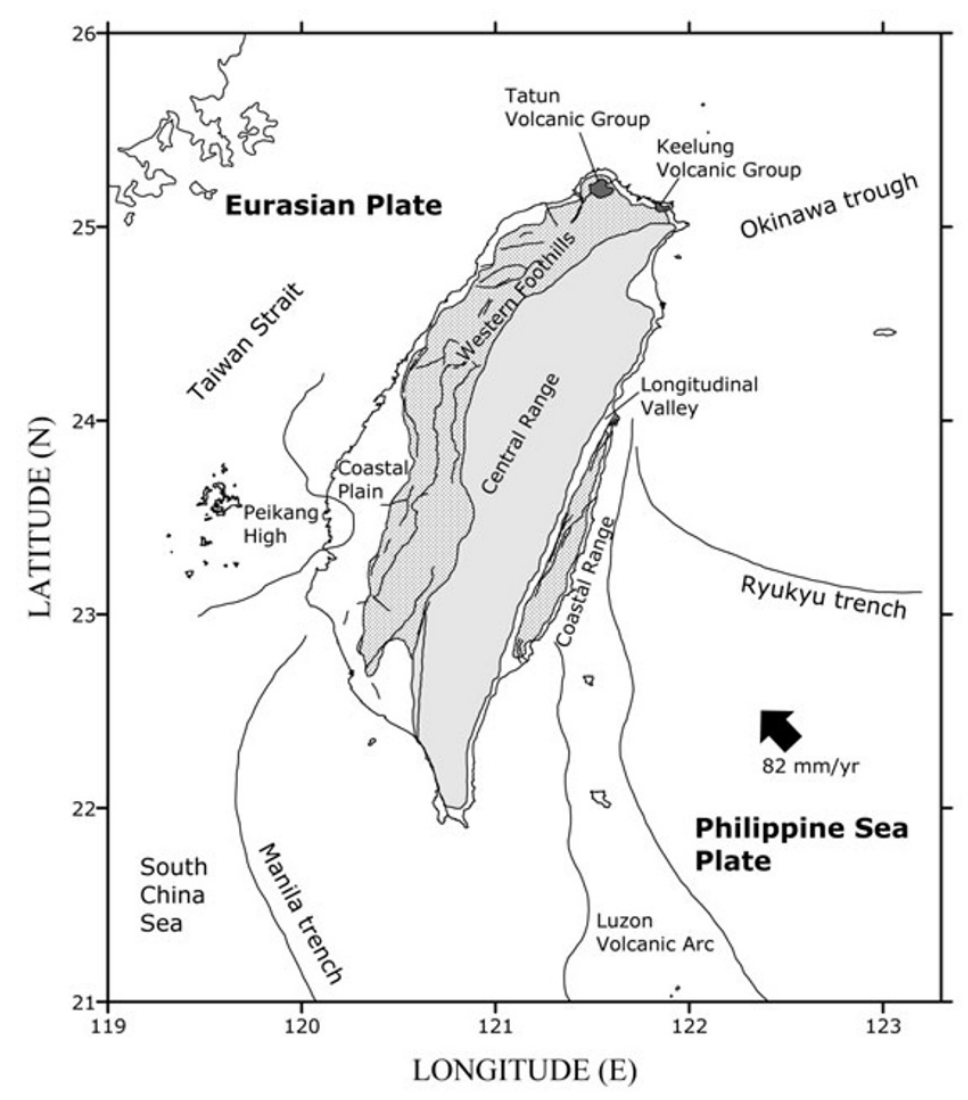

Fig. 5. Simplified map showing the major tectonostratigraphic belts of Taiwan. The foldbelt of the Western Foothills is comprised of the non-metamorphosed Neogene clastic rocks. The Central Range includes the slate formations of the Hsuehshan Range and the Backbone Range, and the higher-grade Paleozoic to Mesozoic metamorphic rocks of the eastern Central Range. The Coastal Range is underlain by Miocene volcanic rocks of andesitic arc magmatism. The segments distributed in the Western Foothills and the Longitudinal Valley represent the Quaternary active faults.

many previous studies in different region of the Earth.

Two horizontal and the vertical components were analyzed for performing the average values, although the estimate of coda $Q$ is independent on the component of wave motion (Del Pezzo and Scarcella, 1986). The sampling rate of digital seismic data used is $100 \mathrm{~Hz}$. All the seismograms were carefully inspected on the computer screen to ensure good quality of the data following the criteria of without spikes, telemetry gaps and multiple signals in the selected coda windows. The overall instrumental response was removed and the baseline drift observed in some seismograms was corrected before the processing of coda $Q$ estimations. Then, the signals were filtered in nine frequency windows with central frequencies of 1.5, 2, 3, 5, 7, 10, 12, 14 and $18 \mathrm{~Hz}$ and with pass band of $0.75 \sim 1.25$ times of central frequency, respectively. Six-pole Butterworth filters with zero phase shifts were used. We calculated smoothed seismic envelopes using the moving window with frequencydependent bandwidth given by $200 \cdot \Delta t / f$, where $\Delta t$ is the sampling interval in seconds. Hence coda $Q$ is determined from the slope of straight line using a least squares fit by $\ln \left(A(f, t) \cdot t^{\alpha}\right)$ versus $t$ over the coda window. Figure 4 represents examples of data processing performed in this study.

Rautian and Khalturin (1978) pointed out that the general form of coda can be established after $2 \sim 3$ times of the $S$-wave travel time since the origin time of event. More- over, the geometry with separated source and receiver as Sato (1977) can be approximated by the one with collocated source and receiver as Aki (1969) after that time. Spudich and Bostwick (1987) have also shown that near-site reverberations can be the dominate component of the coda at lapse time shorter than twice of the $S$-wave travel time, at least for frequencies less than about $10 \mathrm{~Hz}$. The beginning of time window for analyzing coda $Q$ was then chosen as twice the $S$-wave travel time for avoiding contamination from the direct $S$-waves and the site response. The length of coda window generally used herein is $20 \mathrm{sec}$. Both the starting lapse time and the length of coda window can be slightly changed, for obtaining more reliable linear fit, when the abnormal signal is visually recognized to induce a significant interference in the linear regression.

\section{General Tectonic Setting}

Taiwan is located at the junction of two subduction systems, namely, the Ryukyu and Luzon arcs to the east and south, respectively. According to the rapid rate of deformation detected geodetically on the Taiwan region and the high level of seismicity, it is evident that the continuous convergence of northern part of the Luzon arc carried on the Philippine Sea plate and the mainland China continental shelf was responsible for the creation of the island and mountain ranges (Suppe, 1981; Wu et al., 1989; Yu and Chen, 1994) (Fig. 5). The Philippine Sea plate moves to- 
ward Taiwan in the northwest direction and forms an almost south-north linear seismic zone along the eastern Coastal Range. An absolute majority of earthquakes in this linear zone, which is the most active one in Taiwan, are shallower than $40 \mathrm{~km}$ roughly. Events with focal depths less than $25 \mathrm{~km}$, on the other hand, scatter across a wide region from the Taiwan Strait to the Western Foothills and the Central Range. Based on the seismogenic patterns revealed from the broadband waveforms, Kao and Jian (2001) proposed that the subducted Philippine Sea plate beneath northeastern Taiwan, recognized as the slab-continent collision, play a significant role in the overall orogenic process of Taiwan. Due to the collision started in Late Miocene, the orogenic setting mainly comprises two geological provinces separated by the Longitudinal Valley of eastern Taiwan (Ho, 1979). The Coastal Range to the east comprises volcanic and siliciclastic sequences of the accreted Luzon arc system. The Central Range and the Western Foothills to the west consist of metamorphic and sedimentary rocks of deformed China continental margin. As a whole, the basin structural and lithologic units are parallel to the island's NNE-SSW trend and nearly perpendicular to the direction of plate convergence. Moreover, the Tatun and Keelung volcanic groups on land and offshore in northern tip of Taiwan have been interpreted as the western extension of the chain of volcanoes related to the Ryukyu arc system. They appear to result from subduction of the Philippine Sea plate beneath western end of the Ryukyu arc. The clustering of micro-earthquakes with local magnitudes of less than 1 has been monitored recently and indicates that seismic activity is still strong in the Tatun volcanic area (Lin et al., 2005).

\section{Results and Discussions}

Using all the three components of each seismogram recorded from shallow event, the coda $Q$ values were estimated from coda envelopes utilizing the single backscattering method (Aki and Chouet, 1975). For each sector with an included angle of $\pi / 4$, therefore, the average coda $Q$ was obtained from up to three estimates within the corresponding range of station-epicenter azimuth. Figure 6 shows the azimuthal variations of coda $Q$ values in frequency band centered at $5 \mathrm{~Hz}$ at all the used stations. The result has clearly revealed the pattern with no significant dependence on the azimuth herein, even for the frequencies ranging from $1.5 \mathrm{~Hz}$ to $18 \mathrm{~Hz}$ analyzed in this article. It seems to be reasonable as calculating the attenuation rate of coda waves in a similar lapse time for local earthquakes with similar epicentral distance and focal depth.

Coda $Q$ dependence on lapse time has been clearly stated by several workers (Roecker et al., 1982; Pulli, 1984; Canas et al., 1995). Conclusively, the lapse time is mostly related to the region of sampling. According to Pulli (1984), the ellipsoidal volume sampled by coda waves at a time $t$ has an average depth in the relationship: $h=h_{\mathrm{av}}+\left(a^{2}-r^{2} / 4\right)^{1 / 2}$, where $h_{\mathrm{av}}$ is the average focal depth and $r$ is the hypocentral distance. The large semi-axis of surface projection of the ellipsoid having the hypocenter and the station as foci, $a$, can be defined as $v t / 2$, where $v$ is the $S$-wave velocity and can be generally given by $3.6 \mathrm{~km} / \mathrm{sec}$ for the crust of Taiwan area (Chen, 1995). The average lapse time $t$ is represented

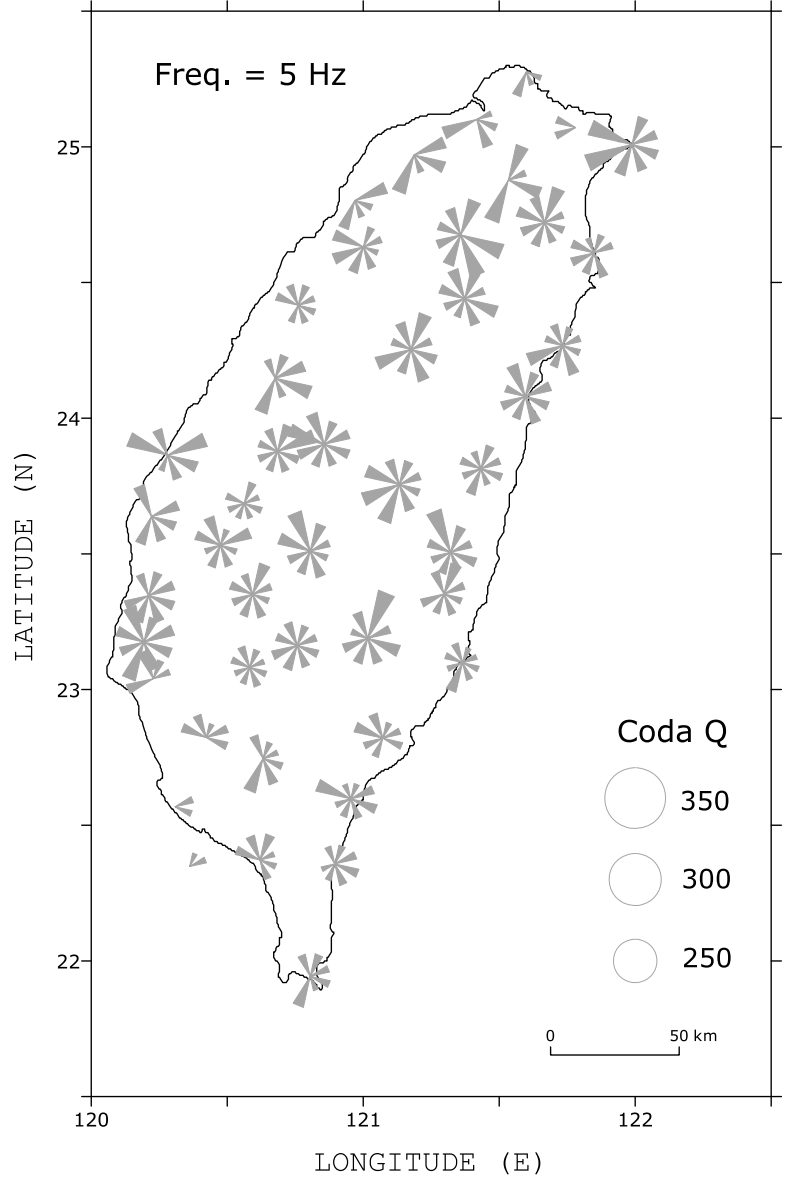

Fig. 6. Azimuthal variation of coda $Q$ value for each station. The length of spreading triangle bar, radiated from the center of each rose map, is proportional to coda $Q$ value scaled to the radii of circles shown in the legends.

by the relationship: $t=t_{\text {start }}+t_{\text {win }} / 2$, where $t_{\text {start }}$ is the starting lapse time and $t_{\text {win }}$ is the window length. Thus, substituting the parameters used into the relationship, the sampled region will be nearly a spheroid with a radius of about $45 \mathrm{~km}$ and centered at the midway position between the station and the hypocenter which are about $20 \mathrm{~km}$ apart. In this situation, the regions characterized by coda $Q$ from different azimuths must be highly overlapped in the vicinity of the station while using very local events.

\section{Frequency-dependent Relationship of Coda $Q$ from Shallow Earthquake Data}

Figure 7 shows all the estimated coda $Q$ values varying from $143 \pm 33$ at $1.5 \mathrm{~Hz}$ to $904 \pm 164$ at $18 \mathrm{~Hz}$. The distribution pattern indicates that the influence of attenuation of the medium decreases with increasing frequency. By using a power-law fitting, an average frequency-dependent coda $Q$ relationship mainly for the crust in Taiwan region is given as following:

$$
Q_{C}=(93 \pm 1) f^{0.75 \pm 0.01}
$$

In previous works for Taiwan region, Chen et al. (1989), using the lapse time of analyzed coda waves visually chosen from 20 to $100 \mathrm{sec}$, proposed a relationship of $Q_{C}=$ $117 f^{0.77}$ which exhibits no obvious variation between the 


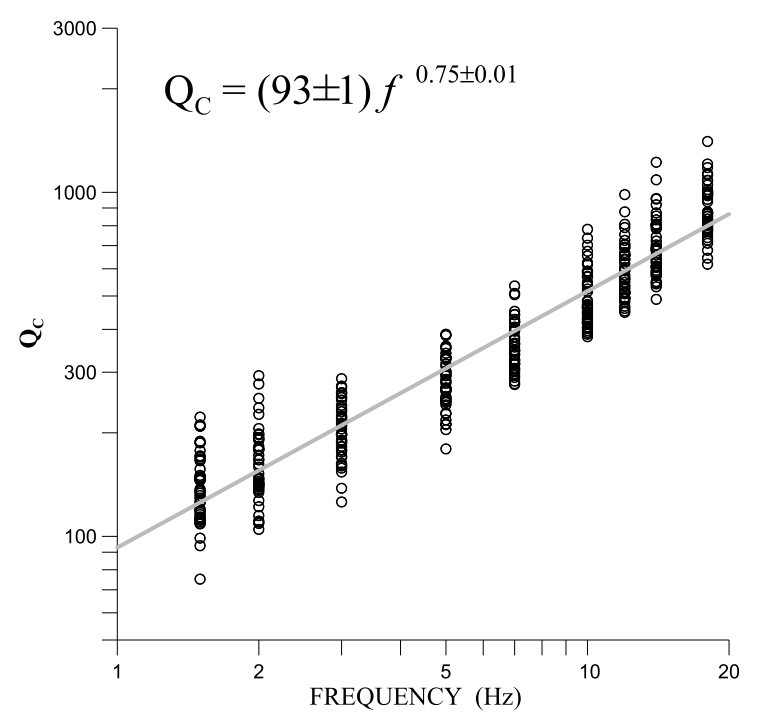

Fig. 7. Map of average coda $Q$ values versus frequency revealed from shallow earthquake data. The corresponding relationship derived by a power-law fit is shown.

eastern and the western Taiwan. An average $Q_{s}$ value of about 200 in frequency ranging from 1 to $6 \mathrm{~Hz}$ for Taiwan region was reported by Wang (1988). The $Q_{s}$ could be equivalent to coda $Q$ (Herrmann, 1980) for comparing with our estimates. In addition, Chen and Nuttli (1984) have derived the $Q_{0}$ of 149 for Taiwan region using instrumental intensity data. Our result, basically, is agreed with theirs.

Reviewing previous coda $Q$ estimations in areas along the border of Pacific Ocean plate, it was found that similar orogenic environment could result in similar seismic-wave attenuation characterized by coda $Q$ value. For example, the coda $Q$ values of range from 80 to 180 at $1 \sim 2 \mathrm{~Hz}$, with a frequency dependence varying from 0.6 to 1.1 , have been derived in Japan area using the data from a dense Hi-net system (Jin and Aki, 2005). From the high-resolution spatial distributions for all of analyzed frequency bands, they pointed out that the low $Q$ zone for $1 \sim 4 \mathrm{~Hz}$ coincides with the zone of high deformation rate revealed from the GPS data, but agrees with volcanic and geothermal region for $4 \sim 16 \mathrm{~Hz}$. It is likely to be concluded both the coda $Q$ and its variation correlated with tectonostratigraphic feature in Japan are similar to those measured in Taiwan. Besides, several studies (Singh and Herrmann, 1983; Xie and Mitchell, 1990; Benz et al., 1997; Baqer and Mitchell, 1998) have consistently found that $Q_{0}$ values, determined from direct $L_{\mathrm{g}}$ and $L_{\mathrm{g}}$ coda of longer lapse time, are in the range of 140 300 for the California coastal region of the United States. Using the control parameters and criteria of event selection comparable to those set in this study, Hellweg et al. (1995) reported 79 for $Q_{0}$ and 0.74 for frequency dependence in Parkfield area, California, which are also in good agreement with our results.

\section{Spatial Distribution of Coda $Q$ from Shallow Earthquake Data}

Averaging over all the available estimates in all the sectors for each station, the spatial distributions of coda $Q$ analyzed by shallow earthquakes data in Taiwan region, for the nine selected frequencies were obtained, respectively. Some of them are shown in Fig. 8. Conspicuous variations of coda $Q$ values are depicted for the studied frequency ranges. The results also show a significant dependence on the frequency. The lower coda $Q$ zone coincides with region from north to south along the backbone of Taiwan Island for frequencies of $1 \sim 2 \mathrm{~Hz}$. It may suggest relatively larger size scatterers in this area, where a great number of historical disastrous earthquakes have occurred during the last century, even though this region does not exhibit the highest level of seismicity. Moreover, the Central Range was produced from the plate collision most probably by extruding the crustal material that also results in a sequence of Quaternary active faults along the Western Foothills. The fractured material within these regions could more rapidly attenuate the coda waves with lower frequencies. In addition, the relatively high coda $Q$ values, for frequencies lower than $5 \mathrm{~Hz}$, along the western coast can be explained by the unapparent attenuation of coda waves scattered from a pre-Miocene basement high, the Peikang High, beneath the Coastal Plain (Tang, 1977; Wang and Shin, 1998; Lin, 2001). Another possible reason could be taken into account anyway. The anomalously high coda $Q$ estimates can be partly attributed to the presence of inefficiently dissipated near surface trapped modes at those stations underlain by the Quaternary sediments (Phillips and Aki, 1986; Wang, 1988). Similar situation seems to be observed at the eastern stations which are located on the Longitudinal Valley, when inspecting the analyzed seismograms. It could be suggested that a further study is necessary for distinguishing the site effects from the observations, since coda excitation had ever been proposed to be dependent on local geology of the site (Aki and Chouet, 1975).

The relative low coda $Q$ zone, however, is shifted toward the east, the south, and the southwest of Taiwan, for frequencies higher than $5 \mathrm{~Hz}$, which coincide well with the seismic zones of clearly recognized (Tsai et al., 1981; Wang and Shin, 1998). As mentioned for the tectonic setting, the Luzon arc, with the underlying Philippine Sea plate, collides the outer continental margin in the Longitudinal Valley paralleled to the eastern coast, while it overrode the Eurasian plate to the south. On the other hand, the preTertiary metamorphic complex of the Central Range, responsible for the low attenuation, represents the unroofed continental basement and probably indicates a relative uniform material sampled by the coda waves in higher frequencies. The general patterns of coda $Q$ distribution in frequency ranging from $3 \mathrm{~Hz}$ to $5 \mathrm{~Hz}$ could be comparatively similar to those of Shin et al. (1987), who used the master curve method (Herrmann, 1980) to deduce coda $Q$ values based on the data from local earthquakes recorded by early CWBSN which only consisted of 15 stations. Using the same data set as used in Shin et al. (1987) and the spectral ratio method, Wang (1988) reported that the largest $Q$ values $(\sim 200)$ for shear waves of $1 \sim 6 \mathrm{~Hz}$ are estimated in the Central Range, while the lower ones of about $110 \sim 150$ along the eastern and western coastal areas and the south Taiwan. The distribution pattern is essentially consistent with ours, excluding in the northwestern area. However, their estimations over the entire Taiwan region are smaller 

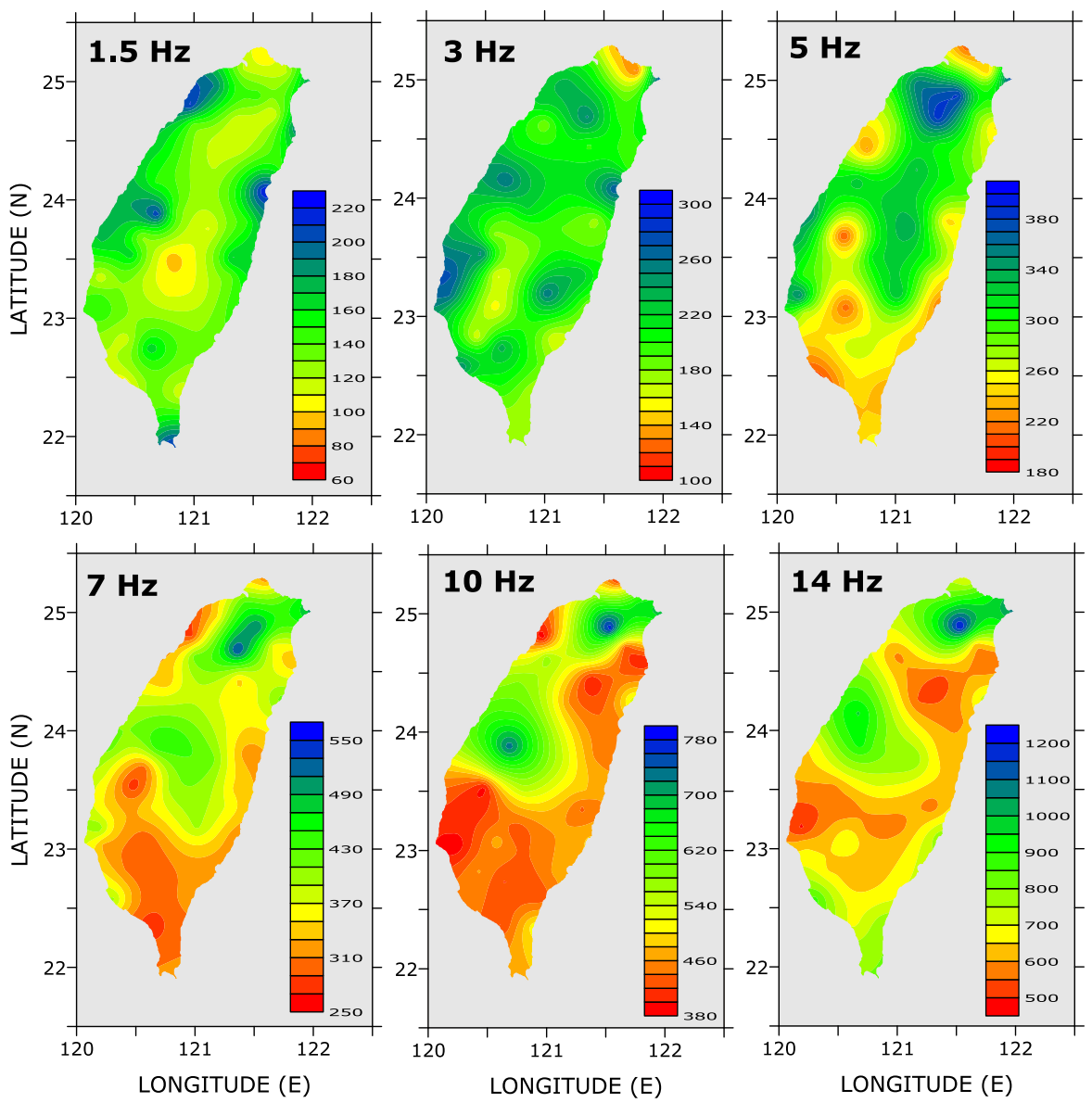

Fig. 8. Spatial distributions of coda $Q$ value for six frequency-bands respectively. The scale of $Q$ value is shown for each subfigure.
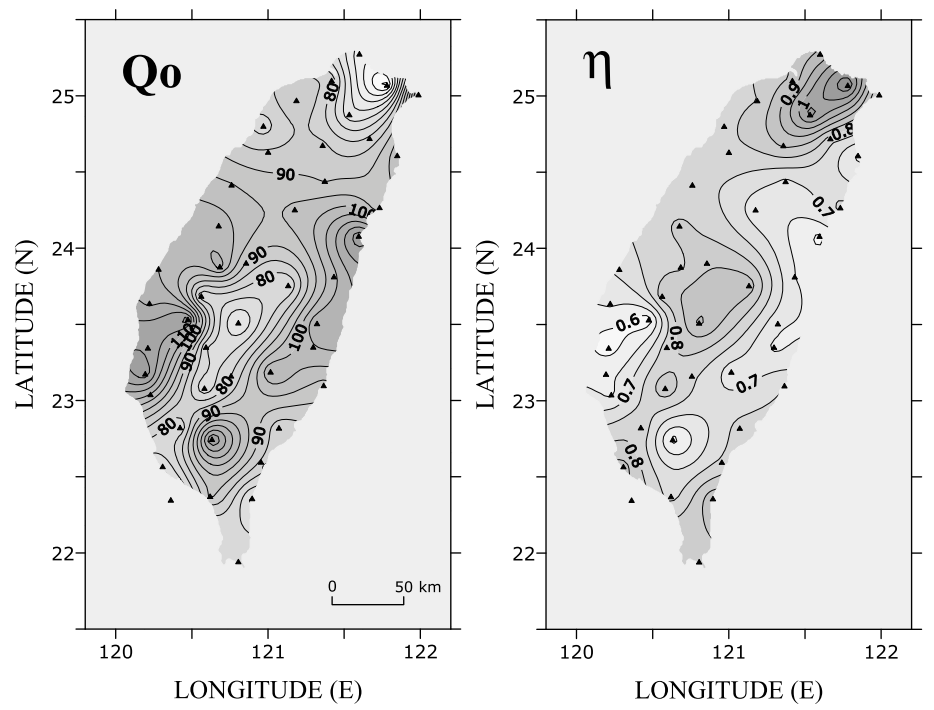

Fig. 9. Spatial distributions of coda $Q$ at frequency of $1 \mathrm{~Hz}\left(Q_{0}\right)$ and the frequency dependence $(\eta)$ estimated from shallow earthquake data. The solid triangles represent the used stations.

than those obtained in this study for the same frequency band, probably due to the utilization of different type of seismic waves. A different result was given by Chen et al. (1989), which presents only a little variation in the spatial distribution of $Q_{0}$ and the frequency dependence, using the lapse time range from 20 to $100 \mathrm{sec}$ for coda waves in the digital recordings from the Taiwan Telemetered Seismo- graphic Network (TTSN). The long lapse time used implies the homogeneity of material at the deeper sampled region.

A distinct low $Q$ zone is obtained in the very tip of north Taiwan. Although limited seismograms were analyzed for these stations, the deviations of $Q$ values were close to the average level for the Taiwan region. Due to the seismicwave paths penetrating through the Tatun and Keelung vol- 

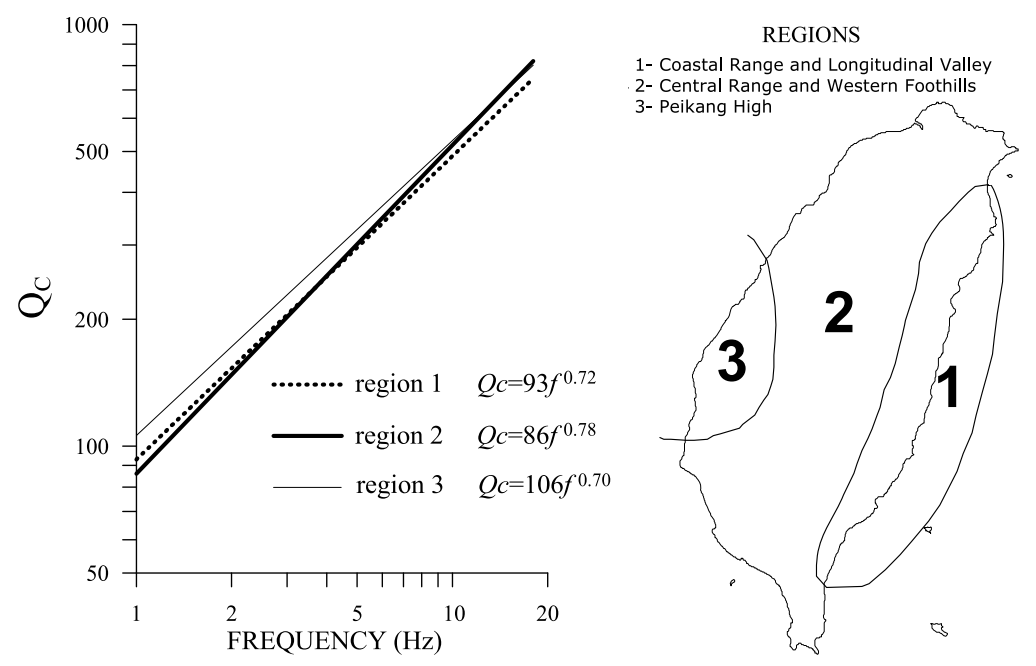

Fig. 10. Comparison between frequency-dependent coda $Q$ values regressed from categorized data, respectively, based on three geological provinces sketched as the right diagram. The $Q_{c}$-frequency relationships are also shown beside the legends.

canic regions, these low $Q$ values may be most possibly explained with the presence of molten materials which are highly heterogeneous. The strong frequency dependence of 0.93 for coda $Q$ values increasing from 94 at $1.5 \mathrm{~Hz}$ to 956 at $18 \mathrm{~Hz}$ can be compared to those observed at volcanic settings around the world (e.g., Canas et al., 1995; Del Pezzo et al., 1995; Gupta et al., 1998; Giampiccolo et al., 2002).

For each station, the frequency-dependent relationship represented by $Q_{C}=Q_{0} f^{\eta}$ can be obtained by the regression analysis. Spatial distributions of coda $Q_{0}$ and frequency dependence $\eta$ are shown in Fig. 9. Coda $Q_{0}$ values are found to be in the range 60 to 120 , in general, and are relative low along the Central Range. The strong frequency dependence ranging from 0.6 to 1.1 identifies the fact of tectonically active region around Taiwan as expected. Comparing the distribution of $Q_{0}$ with that of $\eta$, an inverse correlation between them is likely to be evident over all the region of study. In fact, a better fit for the frequency dependence can be achieved respectively using two different frequency bands separated from about $5 \mathrm{~Hz}$, since larger $\eta$ values could be found at frequency of 5 to $18 \mathrm{~Hz}$ than those at frequency ranging from 1.5 to $5 \mathrm{~Hz}$ for most of stations, especially for the eastern and southern Taiwan. A possible interpretation of the difference in frequency dependence for $1 \sim 5 \mathrm{~Hz}$ and $5 \sim 18 \mathrm{~Hz}$ is that whereas at frequencies lower than $5 \mathrm{~Hz}$ coda $Q$ is largely controlled by intrinsic absorption in the crust, at frequencies higher than $5 \mathrm{~Hz}$ scattering has become more important (Mitchell and Cong, 1998). On the side, the area with most change in frequency dependence of coda $Q$ likely coincides with the plate collision zone, where anomalous heat flow and fractured material definitely exist. It implies that the tectonic setting could be taken into account in resolving this problem, but not in this study.

\section{Coda $Q$ in Various Geologic Provinces}

The frequency dependence of coda $Q$ is usually correlated to the degree of tectonic complexity and heterogeneity of the region under study. Regions of active tectonics where the lithosphere is highly heterogeneous and faulted are characterized by $Q_{C}$ with strong frequency dependence, whereas the stable regions are characterized by $Q_{C}$ with low frequency dependence (Singh and Herrmann, 1983). Utilizing the advantage of high resolution in coda $Q$ spatial distribution derived here, it is allowed to divide Taiwan area into three subregions, mainly based on tectonostratigraphic structure, seismicity, and the $Q_{C}$ distribution patterns, for remodeling the corresponding frequency-dependent relationships respectively. In Fig. 10, the regressive coda $Q$ values respectively for these subgroups are plotted as a function of frequency for comparison. The dotted line indicates the frequency dependence of coda $Q$ with $Q_{0}$ of 93 and $\eta$ of 0.72 , for the region including the Coastal Range and the Longitudinal Valley of eastern Taiwan and extended northward to the northeastern Ilan area. The thin line represents the relationship described with $Q_{0}$ of 106 and $\eta$ of 0.70 , for the restricted area underlain by the Peikang High beneath the western Coastal Plain. Such distinguishable difference between them has definitely explained that seismic waves should exhibit lower decay rate in a stable region than within a seismoactive plate boundary. In an opposite opinion, it is known that oceanic crust (beneath the Coastal Range) normally has a denser composition than continental crust and that energy loss through nonelastic processes decreases with increasing material density and velocity (Lay and Wallace, 1995). Thus, a reasonable explanation to the result is the effect on the attenuation of coda waves performed by fractured rock in the convergence plate boundary (the Longitudinal Valley) is probably larger than the effect attributed to its inherent lithology. However, for most part of regions of the Central Range and the Western Foothills which are placed between the previous two subregions, the strongest frequency dependence, say 0.78 , was obtained and depicted by the thick line in Fig. 10.

\section{Spatial Distribution of Coda $Q$ from Deep Earthquake Data}

For comparison purpose, a set of earthquakes with focal depth ranging from 25 to $50 \mathrm{~km}$ (Fig. 2) were randomly selected without considering azimuth distribution in coda 

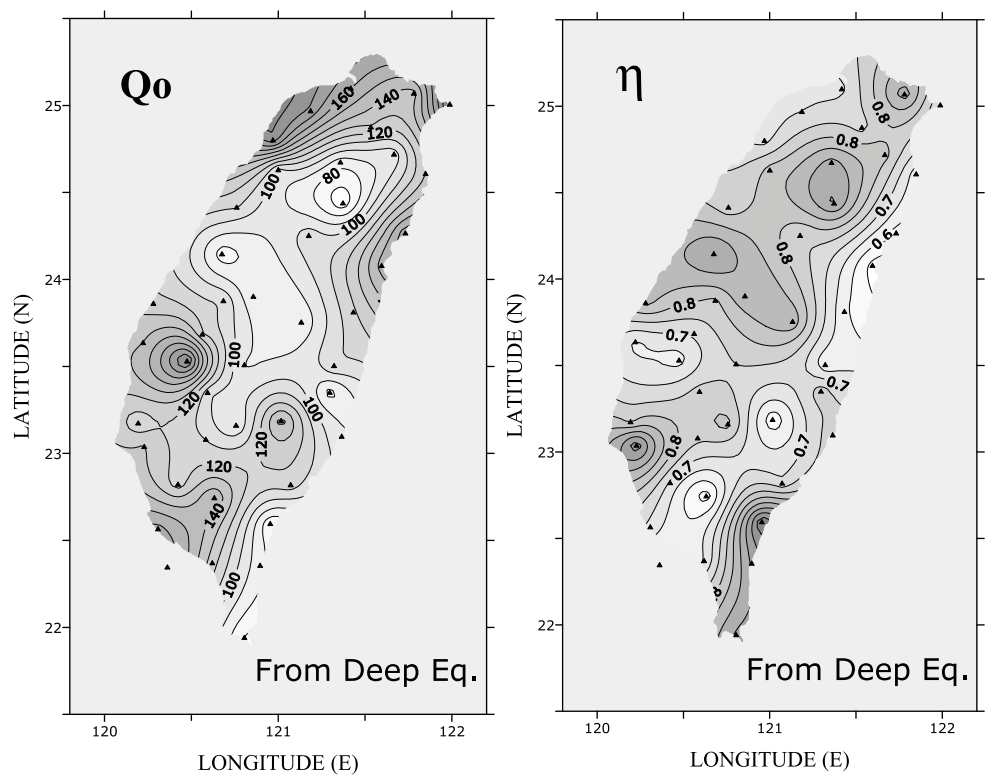

Fig. 11. Spatial distributions of coda $Q$ at frequency of $1 \mathrm{~Hz}\left(Q_{0}\right)$ and the frequency dependence $(\eta)$ estimated from deep earthquake data. The solid triangles represent the used stations.

$Q$ estimations. The criteria in determining critical required parameters are the same as those used in analysis of shallow earthquake data, except the epicentral distances which are mostly shorter than $15 \mathrm{~km}$ to make the hypocenter-tostation connection line approaching to verticality. In this condition, the possible depth sampled by the selected coda waves will reach to about $100 \mathrm{~km}$, based on the previously mentioned formula in evaluating the ellipsoidal volume. Of course, the lapse times of selected coda windows are increased to about $30 \mathrm{sec}$ followed by the criterion of $2 \mathrm{Ts}$, due to the use of larger focal depth. Only maximum of eight recordings from deep events, for each station, were analyzed to obtain the average coda $Q$ values using the same procedures.

After the determination of the power-law coda $Q$ relationship for each station, the spatial distributions of coda $Q_{0}$ and frequency dependence $\eta$ can be described as shown in Fig. 11. Referring to Fig. 9, coda $Q_{0}$ essentially appears a variation pattern similar to that obtained from shallow earthquake data and ranges from 80 to 180 , apparently higher than values associated with the crust. The frequency dependence of coda $Q$, however, varies from 0.6 to 1.0 for most of stations and also remains in a similar distribution pattern with the previous result.

According to similarities in spatial distribution patterns of $Q_{0}$ and $\eta$ between results acquired from two sets of data with different range of focal depth, the regionalizing analysis is once again implemented in this case for more comparisons. The estimated average coda $Q$ values and the corresponding relationships depended on frequency for the three geological provinces defined in Fig. 10 are illustrated in Fig. 12(a)-(c), respectively. For the eastern province consisting of the Coastal Range and the Longitudinal Valley (region 1), the $Q_{0}$ and $\eta$ are estimated to be 109 and 0.70 , respectively, which can be attributed to the fact of existence of highly fractured material. For the central province including the Central Range and the Western Foothills (re- gion 2), the $Q_{0}$ and $\eta$ are increased to 114 and 0.74 , respectively, that can be comparable to the result proposed by Chen et al. (1989). However, a larger standard deviation during the regression analysis for region 2 is inherently obtained due to the larger variation of comprising structures, as the most scattered estimates in frequency band of $1 \sim 3 \mathrm{~Hz}$ revealed in Fig. 12(b). The largest $Q$ values, with $Q_{0}$ of 123 and $\eta$ of 0.76 , were estimated for the region 3 . The relationships shown in Fig. 10, resulted from shallow earthquake data, are also plotted as the dashed lines in the figure. These comparisons explicitly demonstrate that the $Q$ values of lithospheric setting are increasing with the depth. Also, based on the comparison among the $Q-f$ relations of different regions (shown as Fig. 12(d)), it is suggested that the attenuation of later-arriving seismic waves is essentially determined by the macroscopic tectonic feature, whilst the marked crustal heterogeneities may significantly control the attenuation for the early-arriving coda waves observed from very local earthquakes. Finally, the average frequency-dependent coda $Q$ relationship for the upper lithosphere in Taiwan region can be expressed as following:

$$
Q_{C}=(122 \pm 1) f^{0.71 \pm 0.02}
$$

The average coda $Q$ values vary from $180 \pm 67$ at $1.5 \mathrm{~Hz}$ to $1000 \pm 144$ at $18 \mathrm{~Hz}$. Equations (2) and (3), both illustrated in Fig. 12(e), can be generally adopted for the lithosphere with a thickness of about $45 \mathrm{~km}$ and $100 \mathrm{~km}$ as sketched in Fig. 12(f), respectively. Using a rough estimation, the average coda $Q$ values for the upper mantle (in the depth range of $45 \sim 100 \mathrm{~km}$ ) will probably be higher than those of crust by an increment factor of about $50 \%$ in the frequency ranging from $1 \mathrm{~Hz}$ to $18 \mathrm{~Hz}$.

\section{Conclusions}

The detailed crustal $Q_{0}$ distribution could be an important input parameter to the prediction of ground motions for 


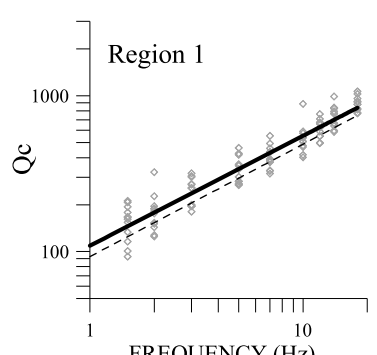

FREQUENCY $(\mathrm{Hz})$

(a)

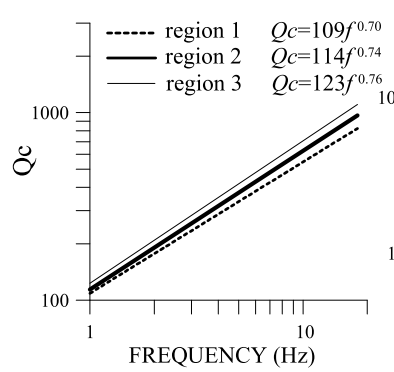

(d)

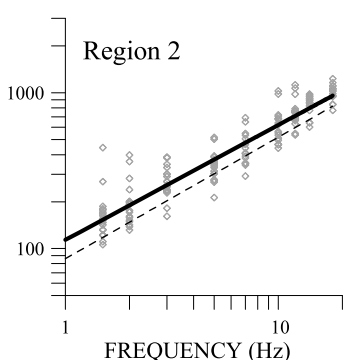

(b)

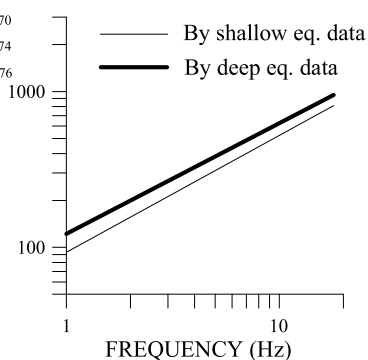

(e)

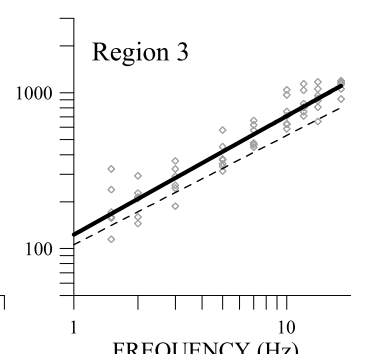

(c)

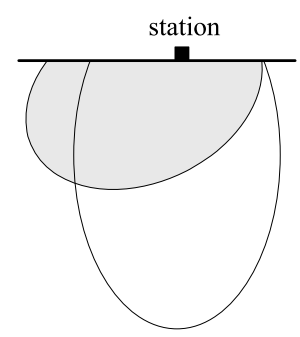

(f)

Fig. 12. Estimated average coda $Q$ (opened diamonds) in studied frequencies and the corresponding relationships (heavy straight lines) for (a) Region 1, (b) Region 2, and (c) Region 3, respectively, which are referred to the zonation map in Fig. 9. The dashed lines shown in (a)-(c) represent the relationships derived from shallow earthquake data for comparisons. Comparison among the relationships from difference tectonic regions is shown in (d). (e) shows relationships for whole Taiwan area by shallow earthquake data (thin line) and deep earthquake data (heavy line), respectively, for which the depth profiles of sampling ellipsoids are sketched as (f).

mapping of isoseismal intensity patterns. In this study, a confirmation of the frequency-dependent relationships for coda $Q$ in Taiwan region have been sought by using the single scattering method (Aki and Chouet, 1975) on shortperiod seismograms collected at the short epicentral distances. The coda $Q$ values, for the frequencies ranging from $1.5 \mathrm{~Hz}$ to $18 \mathrm{~Hz}$, have revealed the pattern with no significant dependence on the propagation direction of direct body waves. However, the spatial distributions are evidently correlated with seismic activity most partly responsible for various tectonic setting. Eventually, the lowest $Q$ values were obtained in the region consisting of the Coastal Range and the Longitudinal Valley of eastern Taiwan, whilst the highest $Q$ values for the region underlain by the Peikang High beneath the western Coastal Plain. These estimations explicitly express that the seismic waves should exhibit lower decay rate in a stable region than within a seismoactive plate boundary. In conclusion, an average frequency-dependent coda $Q$ relationship, $Q_{C}=93 f^{0.75}$, mainly for the crust in Taiwan region was obtained, while $Q_{C}=122 f^{0.71}$ for the upper lithosphere as using deeper earthquake data. Basically, the relations of frequency-dependent coda $Q$ approximately agree with those of previous studies (Shin et al., 1987; Wang, 1988; Chen et al., 1989). Comparing the distributions of $Q_{0}$ and $\eta$, additionally, an inverse correlation between them is definite over all the region of study.

Anomalous coda $Q$ estimates at some stations were observed and reveal apparent inconsistency with the regional levels, when reviewing the spatial distributions as indicated in Fig. 8. To make a deeper investigation concerning the site effects is necessary. The work of Steck et al. (1989) suggested that site effects may dominate the early coda in some regions with resonant structures such as sedimentary basins, variable basement topography, and surface topography, and proposed that the single scattering model is inappropriate in these cases. Several studies (e.g. Phillips and Aki, 1986; Spudich and Bostwick, 1987) have also reported the similar comments. Therefore, due to the station-to-station variation of near-site geological condition attributed to the complexity of tectonic setting in Taiwan, a critical criterion for determining the suitable coda window has to be developed to diminish the effects of possible multiple scatterings, if the single scattering theory is adopted for coda $Q$ estimation using the local earthquake data.

Acknowledgments. We gratefully thank two anonymous reviewers whose constructive suggestions helped us to improve the presentation of this work. We would like to thank the staff of the Earthquake Location Group of Seismology Center, Central Weather Bureau, Taiwan, for their dedicated works on data collection, processing, and archiving to achieve an excellent seismic database. Part of this research was supported by the Central Weather Bureau under grant MOTC-CWB-96-E-09.

\section{References}

Aki, K., Analysis of seismic coda of local earthquakes as scattered waves, J. Geophys. Res., 74, 615-631, 1969.

Aki, K., Attenuation of shear-waves in the lithosphere for frequencies from 0.05 to $25 \mathrm{~Hz}$, Phys. Earth Planet. Inter., 21, 50-60, 1980.

Aki, K., Source and scattering effects on the spectra of small local earthquakes, Bull. Seismol. Soc. Am., 71, 1687-1700, 1981.

Aki, K., Summary of discussions on coda waves at the Istanbul IASPEI meeting, Phys. Earth Planet. Inter., 67, 1-3, 1991.

Aki, K. and B. Chouet, Origin of coda waves: Source, attenuation, and scattering effects, J. Geophys. Res., 80, 3322-3342, 1975.

Baqer, S. and B. J. Mitchell, Regional variation of Lg coda Q in the continental United States and its relation to crustal structure and evolution, Pure Appl. Geophys., 153, 613-638, 1998.

Benz, H. M., A. Frankel, and D. M. Boore, Regional Lg attenuation for the continental United States, Bull. Seismol. Soc. Am., 87, 606-619, 1997.

Canas, J. A., L. G. Pujades, M. J. Blanco, V. Soler, and J. C. Carracedo, Coda-Q distribution in the Canary Islands, Tectonophysics, 246, 245$261,1995$. 
Chang, C. H., Applications of a dense seismic network data on the study of seismogenic structures of central and eastern Taiwan, Ph.D. Thesis, Institute of Geophysics, National Central University, Taiwan, 155 pp, 2004.

Chang, L. S. and Y. T. Yeh, The Q value of strong ground motion in Taiwan, Bull. Inst. Earth Sci., Academia Sinica, 3, 127-148, 1983.

Chen, P. and O. W. Nuttli, Estimates of magnitudes and short-period wave attenuation of Chinese earthquakes from modified Mercalli intensity data, Bull. Seismol. Soc. Am., 74, 957-968, 1984.

Chen, K. C., T. C. Shin, and J. H. Wang, Estimates of coda Q in Taiwan, Proc. Geol. Soc. China, 32, 339-353, 1989.

Chen, Y. L., Three dimensional velocity structure and kinematic analysis in Taiwan area, Master Thesis, Institute of Geophysics, National Central University, Taiwan, 172 pp, 1995.

Del Pezzo, E. and G. Scarcella, Three-component coda Q in the AbruzziMolise region, central Apennines, Ann. Geophys., 4, 589-592, 1986.

Del Pezzo, E., R. Allotta, and D. Patane, Dependence of Qc (coda Q) on coda duration time interval: Model or depth effect?, Bull. Seismol. Soc. Am., 80, 1028-1033, 1990.

Del Pezzo, E., J. Ibanez, J. Morales, A. Akinci, and R. Maresca, Measurements of intrinsic and scattering seismic attenuation in the crust, Bull. Seismol. Soc. Am., 85, 1373-1380, 1995.

Fehler, M., M. Hoshiba, H. Sato, and K. Obara, Separation of scattering and intrinsic attenuation for the Kanto-Tokai region, Japan, using measurements of S-wave energy versus hypocentral distance, Geophys. J. Int., 108, 787-800, 1992.

Frankel, A. and L. Wennerberg, Energy-flux model of seismic coda: separation of scattering and intrinsic attenuation, Bull. Seismol. Soc. Am., 77, 1223-1251, 1987.

Gao, L. S., L. C. Lee, N. N. Biswas, and K. Aki, Comparison of the effects between single and multiple scattering on coda waves for local earthquakes, Bull. Seismol. Soc. Am., 73, 377-389, 1983.

Giampiccolo, E., G. Tusa, H. Langer, and S. Gresta, Attenuation in southeastern Sicily (Italy) by applying different coda methods, J. Seismol., 6, 487-501, 2002.

Giampiccolo, E., S. Gresta, and F. Rascona, Intrinsic and scattering attenuation from observed seismic codas in Southeastern Sicily (Italy), Phys. Earth Planet. Inter., 145, 55-66, 2004.

Gupta, S. C., S. S. Teotia, S. S. Rai, and N. Gautam, Coda Q estimates in the Koyna region, India, Pure Appl. Geophys., 153, 713-731, 1998.

Gusev, A. A., Vertical profile of turbidity and coda Q, Geophys. J. Int., 123, 665-672, 1995 .

Hellweg, M., P. Spudich, J. B. Fletcher, and L. M. Baker, Stability of coda $\mathrm{Q}$ in the region of Parkfield, California: View from the U. S. Geological Survey Parkfield dense seismograph array, J. Geophys. Res., 100, 2089_ 2102, 1995.

Herrmann, R. B., Q estimates using the coda of local earthquakes, Bull. Seismol. Soc. Am., 70, 447-468, 1980.

Ho, C. S., Outline of tectonic framework of Taiwan: presidential address, Proc. Geol. Soc. China, 22, 3-8, 1979.

Jin, A. and K. Aki, Spatial and temporal correction between coda Q and seismicity in China, Bull. Seismol. Soc. Am., 78, 741-769, 1988.

Jin, A. and K. Aki, High-resolution maps of coda Q in Japan and their interpretation by the brittle-ductile interaction hypothesis, Earth Planets Space, 57, 403-409, 2005.

Kao, H. and P. R. Jian, Seismogenic patterns in the Taiwan region: insights from source parameter inversion of BATS data, Tectonophysics, $\mathbf{3 3 3}$, 179-198, 2001.

Kumar, N., I. A. Parvez, and H. S. Virk, Estimation of coda wave attenuation for NW Himalayan region using local earthquakes, Phys. Earth Planet. Inter., 151, 243-258, 2005.

Lay, T. and T. C. Wallace, Modern Global Seismology, Academic Press, $521 \mathrm{pp}, 1995$.

Lee, W. H. K., K. Aki, B. Chouet, P. Johnson, S. Marks, J. T. Newberry, A. S. Ryall, S. W. Stewart, and D. M. Tottingham, A preliminary study of coda Q in California and Nevada, Bull. Seismol. Soc. Am., 76, 11431150,1986

Lin, C. H., The 1999 Taiwan earthquake: A proposed stress-focusing, hellshaped model, Bull. Seismol. Soc. Am., 91, 1053-1061, 2001.

Lin, C. H., K. I. Konstantinou, H. C. Pu, C. C. Hsu, Y. M. Lin, S. H. You, and Y. P. Huang, Preliminary results from seismic monitoring at the Tatun volcanic area of northern Taiwan, Terr. Atmos. Ocean. Sci., 16, 563-577, 2005.

Mayeda, K., S. Koyanagi, M. Hoshiba, K. Aki, and Y. Zeng, A comparative study of scattering, intrinsic, and coda $\mathrm{Q}^{-1}$ for Hawaii, Long Valley and central California between 1.5 and $15 \mathrm{~Hz}$, J. Geophys. Res., 97, 6643$6659,1992$.
Mitchell, B., Regional variation and frequency dependence of $Q_{\beta}$ in the crust of the United States, Bull. Seismol. Soc. Am., 71, 1531-1538, 1981.

Mitchell, B. J. and L. Cong, Lg coda Q and its relation to the structure and evolution of continents: A global perspective, Pure Appl. Geophys., 153, 655-663, 1998.

Phillips, W. S. and K. Aki, Site amplification of coda waves from local earthquakes in Central California, Bull. Seismol. Soc. Am., 76, 627-648, 1986.

Pulli, J. J., Attenuation of coda waves in New England, Bull. Seismol. Soc. Am., 74, 1149-1166, 1984.

Rautian, T. G. and V. I. Khalturin, The use of the coda for determination of the earthquake source spectrum, Bull. Seismol. Soc. Am., 68, 923-948, 1978

Rhea, S., Q determined from local earthquakes in the south Carolina coastal plain, Bull. Seismol. Soc. Am., 74, 2257-2268, 1984.

Roecker, S. W., B. Tucker, J. King, and D. Hatzfeld, Estimates of Q in central Asia as a function of frequency and depth using the coda of locally recorded earthquakes, Bull. Seismol. Soc. Am., 72, 129-149, 1982

Sarker, G. and G. A. Abers, Comparison of seismic body wave and coda wave measure of Q, Pure Appl. Geophys., 153, 665-684, 1998.

Sato, H., Energy propagation including scattering effects: Single isotropic scattering approximation, J. Phys. Earth, 25, 27-41, 1977.

Sato, H., Fractal interpretation of the linear relation between logarithms of maximum amplitude and hypocentral distance, Geophys. Res. Lett., 15, 373-375, 1988.

Sato, H., Multiple isotropic scattering model including P-S conversions for the seismogram envelope formation, Geophys. J. Int., 117, 487-494, 1994

Shin, T. C., W. J. Su, and P. L. Leu, Coda-Q estimates for the Taiwan area, Bull. Geophys. Natl. Central Univ., 27, 111-118, 1987.

Singh, S. and R. B. Herrmann, Regionalization of crustal coda Q in the continental United States, J. Geophys. Res., 88, 527-538, 1983.

Spudich, P. and T. Bostwick, Studies of the seismic coda using an earthquake cluster as a deeply buried seismograph array, J. Geophys. Res. 92, 10526-10546, 1987.

Steck, L. K., W. A. Prothero, and J. Scheimer, Site-dependent coda Q at Mono Craters, California, Bull. Seismol. Soc. Am., 79, 1559-1574, 1989.

Suppe, J., Mechanics of mountain building and metamorphism in Taiwan, Mem. Geol. Soc. China, 4, 67-89, 1981.

Tang, C. H., Late Miocene erosional unconformity on the subsurface Peikang High beneath the Chiayi-Yunlin, Coastal Plain, Taiwan, Mem. Geol. Soc. China, 2, 155-167, 1977.

Tripathi, J. N. and A. Ugalde, Regional estimation of Q from seismic coda observations by the Gauribidanur seismic array (southern India), Phys. Earth Planet. Inter., 145, 115-126, 2004.

Tsai, Y. B., Z. S. Liaw, and T. Q. Lee, A statistical study of the Taiwan telemetered seismographic network data during 1973-1979, Bull. Inst. Earth Sci., Academia Sinica, 1, 1-22, 1981.

Wang, C. Y., Calculations of $\mathrm{Q}_{\mathrm{S}}$ and $\mathrm{Q}_{\mathrm{P}}$ using the spectral ratio method in the Taiwan area, Proc. Geol. Soc. China, 31, 81-89, 1988.

Wang, C. Y. and T. C. Shin, Illustrating 100 years of Taiwan seismicity, Terr. Atmos. Ocean. Sci., 9, 589-614, 1998.

Wu, F. T., K. C. Chen, J. H. Wang, R. McCaffrey, and D. Salzberg, Focal mechanisms of recent large earthquakes and the nature of faulting in the Longitudinal Valley of eastern Taiwan, Proc. Geol. Soc. China, 32, 157-177, 1989.

Wu, R. S., Multiple scattering and energy transfer of seismic waves: separation of scattering effect from intrinsic attenuation-I. Theoretical modeling, Geophys. J. R. Astron. Soc., 82, 57-80, 1985.

Xie, J. and B. J. Mitchell, Attenuation of multiphase surface waves in the Basin and Range Province, Part I: Lg and Lg coda, Geophys. J. Int., 102, 121-137, 1990.

Yu, S. B. and H. Y. Chen, Global positioning system measurements of crustal deformation in the Taiwan arc-continent collision zone, Terr. Atmos. Ocean Sci., 5, 477-498, 1994.

Yun, S., W. S. Lee, K. Lee, and M. H. Noh, Spatial distribution of coda Q in south Korea, Bull. Seismol. Soc. Am., 97, 1012-1018, 2007.

Zeng, Y., F. Su, and K. Aki, Scattering wave energy propagation in a random isotropic scattering medium, 1. Theory, J. Geophys. Res., 96, $607-619,1991$

J.-K. Chung (e-mail: jkchung@cyu.edu.tw), Y.-L. Chen, and T.-C. Shin 